4

7

19

\title{
biosynthetic megacomplex
}

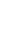

Darren C. Gay*, Drew T. Wagner*, Jessica L. Meinke, Charles E. Zogzas, Glen R. Gay

(1)

\author{
\& Adrian T. Keatinge-Clay
}

Department of Molecular Biosciences, the University of Texas at Austin, 1 University Station A5300, Austin, TX 78712-0165

1

2

3

4

5

6

\section{7}

8

0

21

2

*These authors contributed equally to this work. 


\section{ABSTRACT}

26 Polyketides such as the clinically-valuable antibacterial agent mupirocin are constructed by

27 architecturally-sophisticated assembly lines known as trans-acyltransferase polyketide synthases.

28 Organelle-sized megacomplexes composed of several copies of trans-acyltransferase polyketide

29 synthase assembly lines have been observed by others through transmission electron microscopy

30 to be located at the Bacillus subtilis plasma membrane, where the synthesis and export of the

31 antibacterial polyketide bacillaene takes place. In this work we analyze ten crystal structures of 32 trans-acyltransferase polyketide synthases ketosynthase domains, seven of which are reported

33 here for the first time, to characterize a motif capable of zippering assembly lines into a 34 megacomplex. While each of the three-helix LINKS (Laterally-INteracting Ketosynthase 35 Sequence) motifs is observed to similarly dock with a spatially-reversed copy of itself through 36 hydrophobic and ionic interactions, the amino acid sequences of this motif are not conserved. 37 Such a code is appropriate for mediating homotypic contacts between assembly lines to ensure the ordered self-assembly of a noncovalent, yet tightly-knit, enzymatic network. LINKS39 mediated lateral interactions would also have the effect of bolstering the vertical association of the polypeptides that comprise a polyketide synthase assembly line. 


\section{INTRODUCTION}

Complex polyketides are a class of secondary metabolites that provide a competitive

49

50

51

52

53

54 55 architecture ${ }^{3-5}$. advantage for a wide spectrum of bacteria and fungi, often by targeting vital processes within competing organisms ${ }^{1,2}$. The polyketide synthases (PKSs) responsible for their production are inherently modular, enabling PKS-harboring organisms to explore vast regions of chemical space through the reorganization of enzymatic domains. While the clinical value of the antibiotics erythromycin and mupirocin allude to the potential of polyketides in drug discovery, harnessing the biosynthetic capabilities of PKSs has been hindered by the complexity of their higher-order

An enormous molecular assembly-line of the subcellular milieu, a PKS typically contains upwards of 50 independently-folded domains, each responsible for one step in the biosynthesis of a polyketide. The enzymatic modules of PKSs work together in assembly-line fashion to extend and process a growing polyketide chain ${ }^{6}$, each module containing a set of domains responsible for the incorporation and tailoring of a selected $\alpha$-carboxylated extender unit. The domains employed by Type I PKSs are homologous to those responsible for fatty acid biosynthesis $^{7}$, including acyltransferases (ATs) that select extender units, ketosynthases (KSs) that condense extender units with growing polyketides in a decarboxylative thio-Claisen condensation, ketoreductases (KRs) that stereospecifically reduce newly incorporated $\beta$-keto groups, dehydratases (DHs) that eliminate water to form $\alpha, \beta$-olefins, enoylreductases (ERs) that stereospecifically reduce those olefins, and acyl carrier proteins (ACPs) that shuttle polyketide intermediates between the domains within a module as well as the following module. Thus, modules containing different sets of $\mathrm{KR}$, DH, or ER domains yield different functional groups 
and stereochemistries. A thioesterase (TE) downstream of the final module commonly off-loads the polyketide product via hydrolysis or cyclization.

Modular PKSs are divided into two architecturally-distinct classes: cis-AT and trans$\mathrm{AT}^{2}$. While cis-AT PKSs harbor ATs that are integrated into the multi-domain polypeptide, trans-AT systems rely on discretely-encoded AT domains that noncovalently interact with the megasynthase (Figure 1). Each of the independently-folded domains from cis-AT PKSs has been structurally characterized ${ }^{4}$, and although structural information has recently become available for domains from trans-AT PKSs, if and how the eponymous trans-AT domains dock to the megasynthase to charge ACPs with extender units remains to be determined ${ }^{8-10}$. A conserved 100-residue region C-terminal to KS was hypothesized to facilitate the docking of trans-ATs to megasynthases and was named the AT-docking domain, or ATd ${ }^{11}$. The KS/AT adapters, observed in the crystal structures of KS+AT didomains from the erythromycin PKS and the mammalian fatty acid synthase (FAS), are homologous to ATd on a sequence level and seem to validate this functional assignment ${ }^{12,13}$. However, in vitro experiments examining extender unit transfer from trans-ATs to ACPs showed that the inclusion of ATd in constructs decreased the catalytic efficiency of acyl transfer compared to constructs that did not include ATd $^{14}$.

The first-discovered, and prototypical, trans-AT PKS is encoded within the Bacillus subtilis and Bacillus amyloliquefaciens genomes (pksX and bae, respectively) ${ }^{2}$. This hybrid PKS/non-ribosomal peptide synthetase (NRPS) produces bacillaene, a linear polyene diamide that interferes with prokaryotic protein synthesis (Figure 1) ${ }^{15}$. Transmission electron micrographs of $B$. subtilis cells have revealed dense masses associated with the plasma membrane, each comprised of several copies of the PksX/Bae megasynthase ${ }^{16,17}$. This organelle-sized structure, with an approximate mass of 10-100 MDa, includes the five multidomain polypeptides PksJ, 
92 PksL, PksM, PksN, and PksR. At the time of this discovery megacomplex formation was a 93 complete mystery since trans-AT PKS polypeptides lack the N- and C-terminal docking domains 94 that linearly organize cis-AT PKSs and no PKS architectural scaffolding proteins were known; 95 however, ATd was hypothesized to help stabilize the observed structure (Figure 1) ${ }^{18}$. Membrane localization of PKS enzymes has been observed for the mycolactone and actinorhodin pathways, 97 and the biosynthetic machinery for the siderophore pyoverdine is known to accrete into a dense,

98

membrane-bound mass termed the "siderosome"; the co-localization of large enzymatic complexes at the bacterial plasma membrane is emerging as a theme in the biosynthesis of natural products ${ }^{19-23}$.

The first ATd to be structurally observed, from a didomain composed of a KS and a $\beta$ branching enzyme $(\mathrm{KS}+\mathrm{B})$ from the rhizoxin $\mathrm{PKS}$, revealed that $\mathrm{ATd}$ is structurally related to the KS/AT adapter of $c$ is-AT PKSs ${ }^{8}$. Where the AT domain branches from the KS/AT adapter in cis-AT PKSs, only a short loop is present. The second structure of an ATd, from the second KS domain of the B. subtilis PksX synthase (PksKS2), showed two additional $\alpha$-helices where the AT domain branches from the KS/AT adapter in cis-AT PKSs ${ }^{9}$. Modeling a trans-AT docked to the ATd based on the location of AT within cis-AT PKSs indicated that significant restructuring of these helices would be required for docking. Since neither functional nor structural studies supported the role of ATd as a trans-AT docking site, it was renamed the "flanking subdomain." Comparisons of solved trans-AT KS domains have not yet revealed its role. ${ }^{24}$

Here, we present seven additional crystal structures of trans-AT KS domains that provide evidence that the flanking subdomain plays a role in the assembly of PKS megacomplexes. At first considered to be a serendipitous crystal contact, the clearly defined self-association of flanking subdomains published here and elsewhere appears in ten crystal structures (five 
115 different KS domains from three different microbial species harboring trans-AT PKSs; seven 116 structures reported here, two structures reported in reference 9, and one reported in reference 24).

117 We introduce the term Laterally-INteracting Ketosynthase Sequences (LINKS) to distinguish the 118 three helices that form the observed interaction from the remainder of the flanking subdomain. 119 Bioinformatic analysis of LINKS regions reveals that each is unique to the KS domain 120 possessing them and indicates that PKS assembly lines that form megacomplexes are zippered 121 together by LINKS-containing KSs that possess higher affinity for copies of themselves than 122 other LINKS-containing KSs within the assembly line. These findings suggest an unprecedented 123 mechanism for the assembly of several copies of a PKS megasynthase into a megacomplex.

\section{$125 \quad$ RESULTS}

Structure of the LINKS interaction

Three of the trans-AT KS domains deposited into the Protein Data Bank (PDB) prior to 128 this work contain the LINKS region: one from the bacillaene PKS (PksKS2, PDBs 4NA1 and 129 4NA2) and two from the migrastatin PKS (MgsKS5 and MgsKS7, PDBs 4TL2 and 4TKT, 130 respectively). The LINKS interaction between homodimeric KSs, not apparent from the 131 asymmetric units of wild-type PksKS2 (PDB 4NA1) and MgsKS5 (PDB 4TL2), can be observed 132 by generating the crystallographic symmetry mates. This is opposite in crystals of 133 PksKS2(Cys176Ser) (PDB: 4NA2), in which the asymmetric unit shows two KS monomers 134 associated via the LINKS interaction and the $\mathrm{KS}$ dimer is visualized by generating 135 crystallographic symmetry mates. Here we report seven additional crystal structures in which 136 LINKS interactions are observed, including one of PksKS2 in its monomeric form, one of 137 PksKS6, two of PksKS6(Cys169Ser), one of Pks(ACP5+KS6), one of BaeKS1, and one of 
BaeKS5 (Table 1). To date, only one crystal structure of a LINKS-containing trans-AT KS has been reported that does not show this interaction (MgsKS7, PDB: 4TKT).

The LINKS motif is comprised of $\sim 40$ residues $(\alpha 17-\alpha 19)$ that project from the flanking subdomain as a triangular flap (see reference 9 for secondary structure assignments) (Figure 2). While $\alpha 19$ is structurally equivalent to a helix in the KS/AT adapter of cis-AT PKSs and the mammalian FAS, $\alpha 17$ and $\alpha 18$ take the place of the AT domain in these synthases. Helices $\alpha 17$ and $\alpha 18$ do not make interactions with neighboring secondary structural elements and thus are not rigidly oriented with respect to the flanking subdomain. In the LINKS interaction, $\alpha 18$ of one flanking subdomain makes contact with a spatially-reversed $\alpha 18$ of the neighboring flanking subdomain, and $\alpha 17$ of one flanking subdomain makes contact with $\alpha 19$ of the neighboring flanking subdomain (a dynamic exploration of the interaction is provided in Supplementary Movie 1).

The observed LINKS interactions form through the burial of hydrophobic residues and exhibit a high degree of shape complementarity $\left(\sim 700 \AA^{2}\right)$. In the LINKS motif of PksKS2 (the structure possessing the highest-resolution and lowest B-factors for the LINKS region) these hydrophobic residues include I552, F553, M559, L563, W566, and L578 (Figures 2b-d, and Table S1). The LINKS interactions observed from bacillaene synthases (PksKS2, PksKS6, BaeKS1, and BaeKS5) show contact between clusters of charged residues that cap the poles of $\alpha 18$. The N-terminal end of this helix contains $\sim 3$ negatively-charged residues, and the $\mathrm{C}$ terminal end contains $\sim 3$ positively-charged residues. Two copies of $\alpha 18$ are oriented in an antiparallel fashion, and the negatively-charged $\mathrm{N}$-terminal end of $\alpha 18$ from one flanking subdomain forms salt bridges with the positively-charged C-terminal end of $\alpha 18$ from the neighboring flanking subdomain (Figure S1). Collectively, the burial of exposed hydrophobic 
161 residues in the center of each LINKS triangular flap and the clustering of complementary ionic 162 interactions at both termini of $\alpha 18$ form a lock-and-key fit uniquely adapted to each LINKS 163 interaction (Figure S3).

164 The LINKS motifs connect neighboring KS dimers in crystals of MgsKS5 (PDB: 4TL2);

165 however, instead of the twofold axes of these KS dimers being parallel as in each of the other 166 observed LINKS-mediated associations of KS dimers, they are nearly orthogonal (Figure 2a). 167 The MgsKS5 LINKS interaction is distorted relative to all the other crystallographically168 observed LINKS interactions - $\alpha 17$ and $\alpha 18$ are skewed, and several ionic interactions potentially 169 made in vivo are not formed (e.g., Asp1121/Arg1133 and Glu1122/Arg1129) (Figure S2). The 170 only intact salt bridge, formed by Lys1120 and Asp1138, may provide an example of covariation 171 within the LINKS motif, as both of these residues generally carry the opposite charge in 172 sequence alignments (Figure 3 and Table S2).

173 The seven crystal structures reported here show the LINKS interaction in seven different 174 contexts. In one crystal form, PksKS2 monomers that do not make the traditional KS homodimer 175 interaction were observed. Remarkably, even though $\sim 5300 \AA^{2}$ is not buried through the 176 formation of the traditional KS homodimer, the LINKS interaction is maintained (Figure 2a). 177 The construct Pks(ACP5+KS6), generated to investigate the interaction between KS and ACP, 178 also yielded crystals in which the LINKS interaction was observed. Although the crystals could 179 not be optimized to diffract beyond 4.0- $\AA$ resolution and the ACP domain could not be located in 180 the electron density map, the LINKS interaction was observed to be formed by each of the eight 181 KS monomers in the asymmetric unit (Figure S4). In addition to the $2.0 \AA$-resolution structure of 182 PksKS6(Cys169Ser), a construct generated to visualize bound intermediates ${ }^{9}$, two additional 183 crystal forms of PksKS6 were observed that contain the LINKS interaction (wild-type PksKS6, 
184

185

186

187

188

189

190

191

192

193

194

195

196

197

198

199

200

201

202

203

204

205

206

3.10- $\AA$ resolution; PksKS6(Cys169Ser), 2.16- $\AA$ resolution) (Table S1). Two crystal structures of KSs from the B. amyloliquefaciens bacillaene PKS, BaeKS1 (2.93 $)$ ) and BaeKS5 (4.20 $)$, were also determined. While locally-elevated temperature factors prevented the complete modeling of $\alpha 17-\alpha 19$ in BaeKS1 (Figure S5), the relative positioning of KSs indicates that each of the flanking subdomains form LINKS interactions.

\section{Bioinformatic analysis of LINKS}

The lack of sequence conservation of $\alpha 17-\alpha 19$ is a hallmark of the LINKS motif. While the LINKS motifs shown in Figure 3 are each quite hydrophobic and contain charged residues at the poles of $\alpha 18$, individual LINKS residues are highly variable (Figures 3 and S6). To further investigate this variability, the sequence conservation of LINKS-containing KS domains was mapped onto a model of a trans-AT KS structure (Figure 4). An alignment of 152 trans-AT KS sequences with clear LINKS regions was generated, and the degree of conservation of each residue was assessed. A moving average calculation was used to determine the mean conservation for short stretches of sequence, such that the value for the conservation of residue $n$ is equal to the average value of conservation for the 11 residues ranging from $n-5$ to $n+5$. A template KS structure was then colored based on this value: red for $0-25 \%$, yellow for $25-50 \%$, green for $50-75 \%$, and blue for $75-100 \%$. While the active site region of KS appears blue and the remainder of the KS body varies between green and yellow, the longest stretch of red (46 residues) is the LINKS region. The next longest stretch of red sequence is only 17 residues and is located on the face of KS that would be adjacent to processing domains such as $\mathrm{DH}, \mathrm{KR}$, and MT. Classes of LINKS motifs other than the one shown in the sequence alignment of Figure 3 likely exist; several LINKS motifs that did not align well with those in Figure 3 are displayed in 
207 Figure S6. Due to the highly variable nature of the LINKS region, it is currently unclear whether 208 these sequences self-associate in the same manner as the more canonical LINKS sequences seen 209 in Figure 3.

LINKS interactions are homotypic

With the exception of MgsKS5, the relative orientation of trans-AT KS dimers connected

213 through a LINKS interaction is essentially identical for all of the crystal structures available to 214 date (Figure 2a). We investigated whether heterotypic LINKS interactions were complementary 215 between KSs from different modules. The six LINKS helices (three helices from each KS 216 monomer) were isolated from the observed LINKS interactions (using the structure with the 217 best-resolved LINKS motif for each KS), each of these were superposed, and new coordinate 218 files were created for the heterodimeric LINKS interactions (i.e., PksKS2 with BaeKS1, PksKS6, 219 or MgsKS5). The simulated interactions were investigated to assess the interfacial contacts. In 220 general, heterotypic LINKS interactions were significantly less favorable than homotypic LINKS 221 interactions, displaying much worse shape complementarity with fewer ionic interactions (Figure 222 S7).

\section{DISCUSSION}

The docking site of trans-ATs had been hypothesized to be generated by an 100-residue

227 subdomain, formerly termed ATd and now referred to as the "flanking subdomain", that is 228 associated with the majority of KSs within trans-AT PKSs. That an AT domain is integrated into 229 the equivalent subdomain of the mammalian FAS and cis-AT PKSs appears to support this role; 
however, this subdomain is common to each of these synthases and was most likely present within an ancestral PKS before cis- and trans-AT PKSs diverged ${ }^{25}$. Thus, the docking site to which trans-ATs dock in trans-AT PKSs should not be expected to be equivalent to where ATs are integrated in cis-AT PKSs. A 40-residue LINKS motif is usually found in trans-AT PKSs where the AT domain is integrated in cis-AT PKSs. In 10 out of 11 crystal structures of constructs containing the LINKS motif it is found mediating a LINKS- interaction, and in 8 of the 10 structures it associates neighboring $\mathrm{KS}$ dimers in a parallel orientation (the exceptions being MgsKS5 and the monomeric form of PksKS2). We propose that the LINKS motif makes lateral interactions that help construct megacomplexes like those observed in $B$. subtilis cells (Figure 5; based on the measured dimensions, one bacillaene megacomplex is comprised of 1020 homodimeric bacillaene PKS assembly lines).

The LINKS hypothesis excludes the site that AT is observed in cis-AT PKSs as the docking site for a trans-AT. While trans-ATs could dock with another region of the flanking subdomain or the KS body, they may only interact with the ACP domains to which they transfer extender units $^{27}$. Studies have shown that PksE, a trans-AT in the PksX bacillaene pathway, is present in concentrations $10-100$ fold greater than the assembly line polypeptides ${ }^{17}$. Additionally, superstoichiometric trans-AT concentrations have a large effect on the rate of polyketide production by the virginiamycin trans-AT PKS ${ }^{27}$. If trans-ATs were stably associated with each trans-AT PKS module, increased trans-AT expression would have little effect. Several condensation-incompetent trans-AT KSs harbor flanking subdomains yet have no need to interact with a trans-AT (e.g., BTKS10, ChiKS18, DszKS10, and OnnKS4). Similarly, several trans-AT KSs that catalyze polyketide chain elongation do not contain a flanking subdomain, yet the corresponding ACPs from these modules must interact with a trans-AT (e.g., ChiKS16, 
LnmKS3, MlnKS8, and DifKS10). Studies have shown that trans-ATs are capable of charging

254 ACP domains in the absence of a KS or flanking subdomain and that a trans-AT from the 255 kirromycin PKS (KirCII) is quite specific for its cognate ACP ${ }^{14,26,28-30}$. This degree of trans-AT 256 specificity for an ACP is surprising if the flanking subdomain or KS body is selective for 257 docking with a particular trans-AT.

The structure of the homodimeric KS+B didomain from the rhizoxin trans-AT PKS 259 shows that the longest dimension of the $\mathrm{B}$ dimer is rotated $90^{\circ}$ relative to the longest dimension 260 of the KS dimer ${ }^{8}$. This orientation, also anticipated for the trans-AT KS+DH didomain based on 261 the structural homology of the B and DH domains, would be compatible with the higher-order 262 architecture of a PKS megacomplex formed through LINKS interactions (Figure 5). While 263 several contacts between the KS and B dimers from a trans-AT PKS fix their relative orientation, 264 the KS and DH dimers of the mammalian FAS rotate freely relative to one another ${ }^{13,32}$. The 265 crystal structure of the mammalian FAS shows no contact between the two dimers (Figure S8), 266 and cryo-electron microscopy studies also report flexibility between the KS and DH dimers.

Even if LINKS motifs do not make high affinity interactions, significant avidity could be 268 generated through the several LINKS motifs interacting along the length of the synthase (Figure 269 S9). Within the megacomplex, the lateral LINKS interactions would have the effect of bolstering 270 vertical interactions, thought to be mediated by the $\mathrm{N}$ - and $\mathrm{C}$-terminal regions of trans-AT PKS 271 polypeptides. The flexibility of the LINKS interaction, illustrated by the MgsKS5 crystal 272 structure, could aid megacomplex formation and maintenance. Many trans-AT PKS assembly 273 lines harbor several LINKS motifs (e.g., the bacillaene PKS contains 12, the chivosazol PKS 274 contains 9, the mupirocin PKS contains 7), such that the dissociation of a single LINKS 275 interaction would have little effect on the stability of the entire megacomplex. LINKS 
276 interactions may be difficult to biophysically characterize using methods other than $\mathrm{x}$-ray

277 crystallography. If the dissociation constant for an individual LINKS interaction is in the

278 millimolar range, it may not be observable through techniques such as small-angle $\mathrm{x}$-ray

279 scattering (SAXS) or analytical ultracentrifugation ${ }^{32}$. To accurately determine the dissociation

280 constant of the LINKS interaction, the natural KS dimerization interface may need to be

281 disrupted by site-directed mutagenesis such that only the LINKS-mediated association of KS

282 monomers is measured.

283 Several structures of trans-AT PKS KS domains have been deposited in the PDB that do 284 not contain a LINKS motif, including the KS+B didomain from the rhizoxin PKS ${ }^{8}$. However, 285 only one reported crystal structure of a LINKS-containing KS does not display the LINKS 286 interaction (MgsKS7). Favorable crystal contacts made elsewhere may preclude this interaction 287 in the same manner that LINKS-mediated crystal contacts within the monomeric crystal structure 288 of PksKS2 are favored over natural homodimer interactions.

289 The prevalence of the LINKS motif within many trans-AT systems suggests that 290 megacomplex formation may be a more common occurrence than is currently recognized. The 291 mode through which LINKS interactions could stabilize trans-AT PKS megacomplexes has not 292 been observed in the architectures of other biosynthetic assemblies. The crystal structures 293 presented here reveal a defined binding interface formed by hydrophobic and ionic interactions 294 mediated by three helices on the surface of the flanking subdomain. Bioinformatic analysis 295 suggests that the LINKS interaction is unique for each KS, such that the in-register, homotypic, 296 lateral interactions made by them also confer stability to the vertical interactions between the 297 assembly lines of the megacomplex through avidity. While trans-AT systems represent an 298 excellent template for the rational engineering of synthetic PKSs for the exploration of new 
medicines, it may be necessary to consider the implications of modifying the LINKS network when genetically relocating modules or domains within a trans-AT PKS megasynthase.

\section{ACKNOWLEDGMENTS}

Instrumentation and technical assistance for crystallographic work were provided by Dr. A. Monzingo and the Macromolecular Crystallography Facility, with financial support from the College of Natural Sciences, the Office of the Executive Vice President and Provost, and the Institute for Cellular and Molecular Biology at the University of Texas at Austin. The Berkeley Center for Structural Biology is supported in part by the National Institutes of Health (NIH), National Institute of General Medical Sciences, and the Howard Hughes Medical Institute. The Advanced Light Source is supported by the Director, Office of Science, Office of Basic Energy Sciences, of the US Department of Energy under contract no. DE-AC02-05CH11231. We thank the NIH (GM106112) and the Welch Foundation (F-1712) for supporting this research.

\section{AUTHOR CONTRIBUTIONS}

D.G. solved and refined or assisted in the solution and refinement of each crystal structure, realized that LINKS mediate megacomplex assembly, wrote the manuscript, and generated the figures. D.W. solved and refined or assisted in the solution and refinement of the crystal structures for PksKS6 and its mutant, BaeKS1, and BaeKS5. J.M., C.Z., and G.G. assisted in the solution and refinement of the BaeKS5, BaeKS1, and PksKS2 crystal structures, respectively. A.K.C. provided experimental and crystallographic insight, and assisted in composing the 
manuscript and figures. All authors contributed to editing the manuscript and figures, and declare

323 no competing financial interests.

\section{FIGURE LEGENDS}

\section{Figure 1. Trans-AT vs. cis-AT PKS architecture.}

327 The modular assembly for the archetypal trans-AT (PksX) and cis-AT (6-dEBS) PKSs are 328 shown, responsible for the biosynthesis of bacillaene and erythromycin, respectively. For each 329 system, the fourth PKS module has been magnified to reveal the organization of domains. 330 Although much of the higher-order architecture remains unknown, the schematics represent 331 hypothetical models based on available crystal structures (Ery(KS+AT)3 (PDB: 2QO3), EryDH4 332 (PDB: 3EL6), Spn(KR+ER)2 (PDB: 3SLK), PksKS2 (PDB: 4NA1), and Rhi(KS+B)11 (PDB: 333 4KC5). The presence of a LINKS contact is shown as lateral red extensions for each of the 334 modules in PksX that harbor this region. $\mathrm{N}$ - and $\mathrm{C}$-terminal docking domains for 6-dEBS are 335 shown as vertical blue extensions. ACP, acyl carrier protein; AL, acyl-CoA ligase; AT, 336 acyltransferase; DH, dehydratase; ECH, enoyl-CoA hydratase; ER, enoylreductase; FSD, 337 flanking subdomain; HCS, HMG-CoA synthase; HYD, hydrolase; KS, ketosynthase; KR, 338 ketoreductase; LD, loading didomain; M, module; NRPS, non-ribosomal peptide synthetase; 339 P450, cytochrome P450; TE, thioesterase.

341 Figure 2. The LINKS interaction.

342 The LINKS interaction that forms between trans-AT KS domains is mediated by three $\alpha$-helices $343(\alpha 17-\alpha 19)$ that bind to a spatially-reversed copy of the same structure. (a) A model for the 344 LINKS interaction between two KS homodimers is magnified, revealing the orientation of the 
345 LINKS helices. The angle above the structures indicates the relative angle between KS 346 monomers forming the interaction (Figure S2). Each individual LINKS sequence is colored blue 347 at the $\mathrm{N}$-terminal end of $\alpha 17$ and red at the C-terminal end of $\alpha 19$, and the N-terminal end of $\alpha 17$ 348 always forms favorable interactions with the C-terminal end of $\alpha 19$ from the neighboring KS $349\left(\alpha 19^{\prime}\right)$. The coloring scheme used for the cartoon model has been repeated in each of the 350 displayed structures to highlight the conservation of the LINKS interface. The structure shown 351 for PksKS2 (monomeric form) reveals two KS monomers that do not form the traditional 352 homodimer interaction; however, the LINKS interaction is crystallographically maintained. The 353 relatively high thermal factors for the LINKS helices in BaeKS1 did not permit the complete 354 construction of $\alpha 17-\alpha 19$ for each of the KS monomers, but the relative orientation of the KS 355 bodies indicates that the LINKS interaction is conserved. (b) The three helices forming the 356 LINKS interaction for PksKS2 are shown in cartoon format (green), and the remainder of this 357 PksKS2 monomer has been hidden for clarity. The neighboring KS forming the complementary 358 LINKS interaction is represented with a transparent surface (cyan). The angle of the image is set 359 from the interior of the flanking subdomain, to reveal the collection of centrally-located 360 hydrophobic residues that would be surface-exposed in the absence of the LINKS interaction. (c) 361 A $90^{\circ}$ rotation of the viewing angle shown in panel B reveals the series of ionic interactions 362 formed at the poles of $\alpha 18$. The aspartate and glutamate residues at the N-terminal end of $\alpha 18$ 363 form well-defined ionic interactions with the lysine residues at the C-terminal end of $\alpha 18$ from 364 the neighboring structure. Met559 of the blue monomer can be observed in the center of the 365 image, extending into the hydrophobic cavity of the green monomer (Figure S1a). (d) The three 366 LINKS helices for PksKS2 have been modeled linearly to reduce the complexity of the image. 367 LINKS contacts between residues ranging from 2.0 - $3.0 \AA$ are shown as black dots, and those 
368 ranging from $3.1-4.0 \AA$ are shown as grey dots. The corresponding sequence for the region is 369 also shown, and residues represented by grey letters have been hidden from the cartoon and do 370 not directly contribute to the LINKS interaction.

\section{Figure 3. Sequence alignment of the LINKS region.}

373 Over 100 trans-AT KS sequences were aligned, and a subset of the alignment is shown. 374 Residues in the alignment have been colored according to the following scheme: red for acidic 375 residues, blue for basic residues, and yellow for hydrophobic residues. Helices $\alpha 17-\alpha 19$ (grey) 376 delineate the boundaries of the LINKS region. If a KS in the alignment has been structurally

377 characterized, the associated PDB identifier is shown in parentheses. Although strict 378 conservation of the LINKS region is poor, the conservation of LINKS attributes are generally 379 maintained (i.e., charged poles of $\alpha 18$ flanked by hydrophobic residues). For those KSs that do 380 not contain LINKS (e.g., RhiKS11 and OzmKS9, bottom of alignment), the available structures 381 do not reveal any crystallographic homotypic interactions mediated by this region. The only 382 structure available that contains LINKS but does not exhibit the crystallographic interaction 383 observed in all other trans-AT KS structures is of MgsKS7. The structure of MgsKS5 reveals the 384 LINKS interaction, yet the LINKS attributes differ from what has been observed in other 385 structures. A seemingly anomalous basic residue at the N-terminal end of $\alpha 18$ (Lys1120) forms 386 an ionic interaction with an acidic residue at the N-terminal end of $\alpha 19$ (Asp1138) that is almost 387 invariably basic in the other sequences (both residue positions are marked with asterisks). 388 Accession numbers for the sequences are available in the supporting information (Table S4). 
391 To determine which regions of trans-AT KSs are the most susceptible to sequence variation, a

392 simple moving average (SMA) calculation was performed based on sequence conservation and

393 residues were colored according to the corresponding quartiles. The five regions that received the

394 highest SMA conservation scores all map to the active site of the KS body (blue), while the

395 lowest scoring region corresponds to the LINKS helices (red). A representative KS structure is

396 shown colored accordingly. N- and C-termini are depicted by cyan and red spheres, respectively,

397 and residue numbering is based on the structure of PksKS2.

\section{Figure 5. Vertical and lateral interactions stabilize the PKS megacomplex.}

400 The electron micrographs observed by Straight et al. revealed a dense mass associated with the

401 membrane of $B$. subtilis cells, identified to consist of numerous copies of the PksX 402 megasynthase ${ }^{17}$. An image from that work is reprinted here, revealing that the observed 403 superstructure measures approximately $150 \times 150 \mathrm{~nm}$. The arrow points to the cell membrane, 404 and the arrowhead points to gold nanoparticles engineered to target PksX proteins. For 405 visualizing how this mass of proteins may self-associate, a diagram shows the assembly line 406 subunits (PksJ, PksL, PksM, PksN, and PksR) interacting vertically through domain-domain 407 interactions, and laterally through the LINKS network. The estimations for megacomplex 408 dimensions that flank the cartoon are calculated based on available crystal structures of 409 individual PKS domains. In the vertical dimension, $175 \mathrm{~nm}$ is represented by 15 PKS modules 410 and 2.5 NRPS modules (10 $\mathrm{nm}$ each). In the lateral dimension, $160 \mathrm{~nm}$ is estimated by the total 411 length of $15 \mathrm{KS}$ homodimers that polymerize through LINKS interactions.

\section{TABLES}




\section{Table 1. Crystallographic Data and Refinement Statistics}

\begin{tabular}{|c|c|c|c|c|c|c|c|}
\hline & $\begin{array}{l}\text { PksKS2 (4NA1) } \\
\text { (monomeric) }\end{array}$ & $\begin{array}{l}\text { PksKS6 (5E5N) } \\
\text { (Cys169Ser) }\end{array}$ & $\begin{array}{c}\text { PksKS6 } \\
\text { (5E } 6 \text { K)(Cys169Ser) }\end{array}$ & $\begin{array}{l}\text { PksKS6 } \\
\text { (5ERF) }\end{array}$ & $\begin{array}{l}\text { Pks(ACP5+KS6) } \\
\quad(\text { (5ENY) }\end{array}$ & $\begin{array}{c}\text { BaeKS1 } \\
(5 \mathrm{ELP})\end{array}$ & $\begin{array}{l}\text { BaeKS5 } \\
\text { (5ERB) }\end{array}$ \\
\hline \multicolumn{8}{|l|}{ Data Collection } \\
\hline Wavelength $(\AA)$ & 0.9999 & 1.0332 & 0.9765 & 0.9765 & 1.0332 & 1.0332 & 1.0332 \\
\hline Space group & $\mathrm{C} 2$ & $\mathrm{P} 2_{1}$ & $\mathrm{P} 2_{1}$ & P 1 & P 1 & P 1 & $\mathrm{P} 2_{1}$ \\
\hline $\begin{array}{l}\text { Cell dimensions, } \\
\text { a, } b, c(\AA) ; \alpha, \beta, \gamma \\
\left({ }^{\circ}\right)\end{array}$ & $\begin{array}{l}197.1,75.1 \\
134.1 ; 90.0 \\
132.9,90.0\end{array}$ & $\begin{array}{c}89.4,108.2 \\
151.2 ; 90.0 \\
96.4,90.0\end{array}$ & $\begin{array}{c}76.7,105.5,89.3 \\
90.0,96.8,90.0\end{array}$ & $\begin{array}{c}69.0,77.3 \\
85.3 ; 77.7 \\
70.3,64.2\end{array}$ & $\begin{array}{c}\text { 63.1, 112.7, } \\
211.4 ; 105.0 \\
90.1,106.3\end{array}$ & $\begin{array}{c}61.2,100.0 \\
100.8 ; 91.9 \\
88.2,96.0\end{array}$ & $\begin{array}{c}70.8,319.1 \\
103.5 ; 90.0 \\
110.3,90.0\end{array}$ \\
\hline Resolution $(\AA)$ & $47.25-3.02$ & $150.89-2.00$ & $36.55-2.16$ & $39.73-3.10$ & $39.77-4.00$ & $99.35-2.93$ & $97.07-4.20$ \\
\hline$R_{\text {merge }}$ & $0.048(0.663)$ & $0.120(0.570)$ & $0.092(0.481)$ & $0.058(0.359)$ & $0.102(0.373)$ & $0.148(0.641)$ & $0.114(0.483)$ \\
\hline$I / \sigma(I)$ & $32.2(1.8)$ & $28.1(2.5)$ & $12.3(2.0)$ & $13.3(1.7)$ & $6.1(2.7)$ & $15.7(1.5)$ & $14.2(2.8)$ \\
\hline Completeness (\%) & $99.7(98.4)$ & $99.2(96.0)$ & $99.2(93.5)$ & $94.1(75.6)$ & $98.7(99.0)$ & $94.9(89.75)$ & $79.9(59.3)$ \\
\hline Redundancy & $3.7(3.8)$ & $2.1(2.1)$ & $1.9(1.8)$ & $1.9(1.9)$ & $3.3(3.5)$ & $1.1(1.1)$ & $3.2(2.8)$ \\
\hline $\begin{array}{l}\text { Wilson B value } \\
\left(\AA^{2}\right)\end{array}$ & 119.7 & 35.3 & 42.5 & 77.3 & 157.2 & 62.4 & 196.4 \\
\hline \multicolumn{8}{|l|}{ Refinement } \\
\hline Resolution $(\AA)$ & $47.25-3.02$ & $150.89-2.00$ & $36.55-2.16$ & $39.73-3.10$ & $39.77-4.00$ & $99.35-2.93$ & $97.07-4.20$ \\
\hline \multirow[t]{2}{*}{ No. of reflections } & & 183336 & & & & & \\
\hline & 26825 (1879) & (13049) & 71285 (4947) & $24128(1403)$ & 39874 (2246) & 46279 (3209) & 23712 (1314) \\
\hline$R_{\text {work }} / R_{\text {free }}$ & $0.223 / 0.268$ & $0.201 / 0.231$ & $0.224 / 0.258$ & $0.218 / 0.262$ & $0.322 / 0.344$ & $0.262 / 0.288$ & $0.288 / 0.341$ \\
\hline \multicolumn{8}{|l|}{ No. of atoms } \\
\hline Protein & 8938 & 17314 & 8463 & 8944 & 34416 & 16904 & 18315 \\
\hline Water & - & 1358 & 88 & - & - & - & - \\
\hline \multicolumn{8}{|l|}{$\begin{array}{l}\text { Average } B \text { factors } \\
\left(\AA^{2}\right)\end{array}$} \\
\hline Protein & 119.7 & 44.3 & 42.7 & 77.8 & 157.3 & 63.1 & 196.4 \\
\hline Water & - & 27 & 42.4 & - & - & - & - \\
\hline \multicolumn{8}{|l|}{ RMS deviations } \\
\hline $\begin{array}{l}\text { Bond lengths } \\
(\AA)\end{array}$ & 0.0018 & 0.0186 & 0.0083 & 0.0180 & 0.0013 & 0.0127 & 0.0130 \\
\hline Bond angles $\left({ }^{\circ}\right)$ & 1.084 & 1.352 & 1.342 & 1.390 & 0.716 & 1.937 & 2.651 \\
\hline \multicolumn{8}{|l|}{$\begin{array}{l}\text { Ramachandran } \\
\text { Statistics (\%) }\end{array}$} \\
\hline $\begin{array}{l}\text { Preferred } \\
\text { Regions }\end{array}$ & 91.8 & 97.3 & 96.5 & 94.0 & 95.8 & 92.6 & 93.3 \\
\hline
\end{tabular}




$\begin{array}{cccccccc}\begin{array}{c}\text { Allowed } \\ \text { Regions }\end{array} & 8.2 & 2.7 & 3.5 & 5.9 & 4.2 & 6.7 & 6.6 \\ \text { Outliers } & 0.0 & 0.0 & 0.0 & 0.1 & 0.0 & 0.7 & 0.1\end{array}$

415

416

417 METHODS model.

All protein structures described in this work were cloned, purified, and the corresponding structures refined using identical methods to those previously described for PksKS2 ${ }^{9}$. Therefore, only the primers used for gene amplification of new constructs, crystallization conditions, phasing methods, and any other modifications will be reported here.

Crystals of PksKS2 in which the KS domains did not crystallize in the native dimeric form grew over a period of 5-7 days by sitting drop vapor diffusion at $4{ }^{\circ} \mathrm{C}$. Drops were formed by mixing $2 \mu \mathrm{L}$ protein solution $(4.5 \mathrm{mg} / \mathrm{ml} \mathrm{PksKS} 2,150 \mathrm{mM} \mathrm{NaCl}, 10 \%$ glycerol, $10 \mathrm{mM}$ HEPES, pH 7.5) with $1 \mu \mathrm{L}$ crystallization buffer (30\% PEG $400(\mathrm{v} / \mathrm{v}), 0.2 \mathrm{M} \mathrm{MgCl}_{2}, 0.1 \mathrm{M}$ HEPES, pH 7.5). Crystals were frozen in liquid nitrogen after a 20 min soak in crystallization buffer modified with $20 \%$ glycerol, and the diffraction data collected at ALS Beamline 5.0.3. The structure was solved to $3.0 \AA$ resolution by molecular replacement with PhaserMR in the CCP4 suite, using a single chain of the dimeric structure of PksKS2 (PDB: 4NA1) as the search

The DNA encoding PksKS6 was amplified using primers: 5'GCGGCCTGGTGCCGCGCGGCTCTAGCGACCGCCCGGAGGATGCGATAG-3' and 5'GTGGTGGTGGTGGTGGTGATGTTAGCCTTCTTGAGTTGGCAGCCAG-3' (LIC cloning regions underlined for insertion into the plasmid pGAY $28^{33}$ ). The eluted protein was concentrated to $15 \mathrm{mg} / \mathrm{mL}$ in the equilibration buffer and stored at $-80{ }^{\circ} \mathrm{C}$ until needed. Crystals of PksKS6 grew in 1-6 days by sitting drop vapor diffusion at $22{ }^{\circ} \mathrm{C}$. Drops were formed by mixing 
$4372 \mu \mathrm{L}$ protein solution (15 mg/mL PksKS6, $150 \mathrm{mM} \mathrm{NaCl}, 10 \mathrm{mM}$ HEPES, pH 7.5) with $1 \mu \mathrm{L}$ 438 crystallization buffer $\left(150 \mathrm{mM}\left(\mathrm{NH}_{4}\right)_{2} \mathrm{SO}_{4}{ }^{-2}, 15 \%\right.$ PEG $4000(\mathrm{v} / \mathrm{v}), 100 \mathrm{mM} \mathrm{MES,} \mathrm{pH} \mathrm{6.0).}$ 439 Crystals were frozen in liquid nitrogen after a 20 min soak in the crystallization buffer modified 440 with $10 \%$ (v/v) ethylene glycol. Diffraction data, collected at ALS Beamline 5.0.3, were 441 processed by HKL2000. The structure was solved to $3.00 \AA$ resolution by molecular replacement 442 using the KS2 monomer from the PksX synthase of B. subtilis as the search model (PDB: 443 4NA1).

The PksKS6(Cys169Ser) expression plasmid was generated by PCR amplification from 445 the PksKS6 construct described above using primers 5'AGACGGCCTCGAGCTCTTCATTGGTCGGCACTCATTTAGCGCGCCAGGCACTTATAA

447 ACAAAG-3' and $5^{\prime}-$ AGAACTGCTCGAGGCGGTATCAATAGGAATCGCTGGCCCTTTTAAATTTAGAAAATA

449 CGG-3' (XhoI site underlined). The PCR product was digested with XhoI and ligated. Crystals 450 of PksKS6(Cys169Ser) grew over 2-10 days by sitting drop vapor diffusion at $22{ }^{\circ} \mathrm{C}$. Drops were 451 formed by mixing $2 \mu \mathrm{L}$ protein solution (15 mg/mL PksKS6(Cys169Ser), $150 \mathrm{mM} \mathrm{NaCl,} 10 \mathrm{mM}$ 452 HEPES, pH 7.5) with $1 \mu \mathrm{L}$ crystallization buffer (1.7 $\mathrm{M} \mathrm{LiSO}_{4}, 100 \mathrm{mM}$ Tris, pH 8.0) (both 453 crystal forms grew in the same crystallization condition). Crystals were frozen in liquid nitrogen 454 after a 20 min soak in crystallization buffer modified with $10 \%(\mathrm{v} / \mathrm{v})$ ethylene glycol and the 455 diffraction data were collected at APS Beamline 23-ID-D. The structure was solved by molecular 456 replacement using a single chain of the dimeric structure of PksKS2 (PDB: 4NA1) as the search 457 model.

Bacillus amyloliquefaciens FZB42 genomic DNA was used as template DNA to PCR 459 amplify BaeKS1 with KAPA High-Fidelity DNA polymerase (KAPA) using the forward primer 

reverse primer 5'- GTGGTGGTGGTGGTGGTGATGTTAGTTCTTTTCTGCTTTTTTCGGCG3' (pGAY28 LIC cloning regions underlined). The eluted protein was concentrated to $14 \mathrm{mg} / \mathrm{mL}$

463 in the equilibration buffer and stored at $-80^{\circ} \mathrm{C}$ until needed. Crystals of BaeKS1 grew over 4-15 464 days by sitting drop vapor diffusion at $22{ }^{\circ} \mathrm{C}$. Drops were formed by mixing $2 \mu \mathrm{L}$ protein 465 solution (14 mg/mL BaeKS1, $150 \mathrm{mM} \mathrm{NaCl,} 10 \mathrm{mM}$ HEPES, pH 7.5) with $1 \mu \mathrm{L}$ crystallization 466 buffer (32\% PEG $400(\mathrm{v} / \mathrm{v}), 0.2 \mathrm{M} \mathrm{MgCl}_{2}, 100 \mathrm{mM}$ HEPES, pH 7.5). Crystals were frozen in 467 liquid nitrogen after a 20 min soak in crystallization buffer modified with 40\% PEG 400 (v/v) 468 and the diffraction data were collected at APS Beamline 23-ID-D. The structure was solved by 469 molecular replacement using a single chain of the dimeric structure of PksKS2 (PDB: 4NA1) as 470 the search model.

The gene corresponding to $\mathrm{Pks}(\mathrm{ACP} 5+\mathrm{KS} 6)$ was PCR amplified using primers 5'472 GCGGCCTGGTGCCGCGCGGCTCTAGCGCTGAAGAAACGATTCAATATGC-3' and 5'473 GTGGTGGTGGTGGTGGTGATGTtAGCCTTCTTGAGTTGGCAGC-3' （pGAY28 LIC 474 cloning regions underlined). Crystals grew over a period of 2-4 days by sitting drop vapor 475 diffusion at $22{ }^{\circ} \mathrm{C}$. Drops were formed by mixing $2 \mu \mathrm{L}$ protein solution $(25 \mathrm{mg} / \mathrm{ml}$ 476 Pks(ACP5+KS6), $150 \mathrm{mM} \mathrm{NaCl,} \mathrm{10 \%} \mathrm{glycerol,} 10 \mathrm{mM}$ HEPES, pH 7.5) with $1 \mu \mathrm{L}$ 477 crystallization buffer (30\% PEG 4000 (v/v), 0.2 M sodium acetate, 0.1 M Tris, pH 8.5). Crystals 478 were frozen in liquid nitrogen after a 20 min soak in crystallization buffer modified with $20 \%$ 479 glycerol, and the diffraction data collected at ALS Beamline 5.0.2. Phases were obtained by 480 molecular replacement using PksKS6 (wild-type) as a search model. The structure was solved to $4814.0 \AA$ A resolution by molecular replacement using a single chain from the dimeric structure of 482 PksKS6 as the search model. Electron density for the eight ACPs in the asymmetric unit could 
483 not be located despite exhaustive inspection of the map.

484 The DNA encoding BaeKS5 was amplified using primers: 5'485 GCGGCCTGGTGCCGCGCGGCTCTAGCCAGCAGCTGACAGAGCGTG-3' and 5'486 GTGGTGGTGGTGGTGGTGATGTTAATCAGCTGATGTGTCGATCCAATAAC 487 (pGAY28 LIC cloning regions underlined). The eluted protein was concentrated to $9.9 \mathrm{mg} / \mathrm{mL}$ in 488 the equilibration buffer. Crystals of BaeKS5 grew in 2 days by sitting drop vapor diffusion at 22 $489{ }^{\circ} \mathrm{C}$. Drops were formed by mixing $1.75 \mu \mathrm{L}$ protein solution $(9.9 \mathrm{mg} / \mathrm{mL}$ BaeKS5, $150 \mathrm{mM} \mathrm{NaCl}$, $49015 \mathrm{mM}$ HEPES, $\mathrm{pH}$ 7.5) with $0.5 \mu \mathrm{L}$ crystallization buffer (1.1 $\mathrm{M} \mathrm{LiSO}_{4}, 0.1 \mathrm{M}$ Tris, $\left.\mathrm{pH} 8.0\right)$. 491 Crystals were frozen in liquid nitrogen after a 20 min soak in the crystallization buffer modified 492 with $10 \%$ (v/v) glycerol. Diffraction data, collected at APS Beamline 23-ID-B, were processed 493 by HKL2000. The structure was solved to $4.20 \AA$ resolution by molecular replacement using the 494 KS2 monomer from the PksX synthase of B. subtilis as the search model (PDB: 4NA1).

\section{REFERENCES}

497

498 1. Hertweck, C. The biosynthetic logic of polyketide diversity. Angew. Chem. Int. Ed. 499 Engl. 48, 4688-716 (2009).

500

501 2. Piel, J. Biosynthesis of polyketides by trans-AT polyketide synthases. Nat. Prod. Rep. 27, 996$5021047(2010)$.

503

504 3. Walsh, C.T. The chemical diversity of natural-product assembly lines. Acc. Chem. Res. 41, 4$50510(2008)$. 
507 4. Keatinge-Clay, A.T. The structures of type I polyketide synthases. Nat. Prod. Rep. 29, 1050$5081073(2012)$.

509

510 5. Dutta, S. et al. Structure of a modular polyketide synthase. Nature 510, 512-517 (2014).

512 6. Khosla, C., Herschlag, D., Cane, D.E., \& Walsh, C.T. Assembly line polyketide synthases: 513 mechanistic insights and unsolved problems. Biochemistry 53, 2875-2883 (2014).

514

515 7. Smith, S. \& Tsai, S.C. The type I fatty acid and polyketide synthases: a tale of two 516 megasynthases. Nat. Prod. Rep. 24, 1041-1072 (2007).

517

518

8. Bretschneider, T. et al. Vinylogous chain branching catalyzed by a dedicated polyketide 519 synthase module. Nature 502, 124-128 (2013).

520

521 9. Gay, D. et al. A close look at a ketosynthase from a trans-acyltransferase modular polyketide 522 synthase. Structure 22, 441-451 (2014).

523

524 10. Piasecki, S.K., Zheng, J., Axelrod, A.J., Detelich, M E., \& Keatinge-Clay, A.T. Structural 525 and functional studies of a trans-acyltransferase polyketide assembly line enzyme that catalyzes 526 stereoselective $\alpha$ - and $\beta$-ketoreduction. Proteins DOI: 10.1002/prot.24561 (2014). 
528 11. Tang, G.-L., Cheng, Y.-Q., \& Shen, B. Leinamycin biosynthesis revealing unprecedented 529 architectural complexity for a hybrid polyketide synthase and nonribosomal peptide synthetase. 530 Chem. Biol. 11, 33-45 (2004).

531

532 12. Tang, Y., Chen, A.Y., Kim, C.Y., Cane, D.E., \& Khosla, C. Structural and mechanistic 533 analysis of protein interactions in module 3 of the 6-deoxyerythronolide B synthase. Chem. Biol. 534 14, 931-943 (2007).

535

536 13. Maier, T., Leibundgut, M., \& Ban, N. The crystal structure of a mammalian fatty acid 537 synthase. Science 321, 1315-1322 (2008).

538

539 14. Aron, Z.D., Fortin, P.D., Calderone, C.T., \& Walsh, C.T. FenF: Servicing the mycosubtilin 540 synthetase assembly line in trans. ChemBioChem 8, 613-616 (2007).

541

542 15. Patel, P.S. et al. Bacillaene, a novel inhibitor of procaryotic protein synthesis produced by 543 Bacillus subtilis: Production, taxonomy, isolation, physico-chemical characterization and 544 biological activity. J. Antibiot. 48, 997-1003 (1995).

545

546 16. Butcher, R.A. et al. The identification of bacillaene, the product of the PksX megacomplex in 547 Bacillus subtilis. Proc. Natl. Acad. Sci. U.S.A. 104, 1506-1509 (2007). 
549 17. Straight, P.D., Fischbach, M.A., Walsh, C.T., Rudner, D.Z., \& Kolter, R. A singular 550 enzymatic megacomplex from Bacillus subtilis. Proc. Natl. Acad. Sci. U.S.A. 104, 305-310 551 (2007).

552

553 18. Broadhurst, R.M., Nietlispach, D., Wheatcroft, M.P., Leadlay, P.F., \& Weissman, K.J. The 554 structure of docking domains in modular polyketide synthases. Chem. Biol. 10, 723-731 (2003). 555

19. Xu, X.-P. et al. Localization of the ActIII actinorhodin polyketide ketoreductase to the cell wall. FEMS Microbiol. Lett. 287, 15-21 (2008).

558

559 20. Imperi, F. \& Visca, P. Subcellular localization of the pyoverdine biogenesis machinery of 560 Pseudomonas aeruginosa: A membrane-associated "siderosome". FEBS Lett. 587, 3387-3391 561 (2013).

562

563 21. Porter, J.L. et al. The cell wall-associated mycolactone polyketide synthases are necessary 564 but not sufficient for mycolactone biosynthesis. PLOS ONE 8(7): e70520 (2013).

565

566 22. Simunovic, V. et al. Myxovirescin A biosynthesis is directed by hybrid polyketide 567 synthases/nonribosomal peptide synthetase, 3-hydroxy-3-methylglutaryl-CoA synthases, and 568 trans-acting acyltransferases. ChemBioChem 7(8), 1206-1220 (2006).

569

570 23. Hantash, F.M. \& Earhart, C.M. Membrane association of the Escherichia coli enterobactin 571 synthase proteins EntB/G, EntE, and EntF. J. Bacteriol. 182, 1768-1773 (2000). 
573 24. Lohman, J.R. et al. Structural and evolutionary relationships of "AT-less" type I polyketide 574 synthase ketosynthases. PNAS 112, 12693-12698 (2015).

575

576 25. Nguyen, T. et al. Exploiting the mosaic structure of trans-acyltransferase polyketide 577 synthases for natural product discovery and pathway dissection. Nat. Biotechnol. 26, 225-233 578 (2008).

579

580 26. Ye, Z., Musiol, E.M., Weber, T., \& Williams, G.J. Reprogramming acyl carrier protein 581 interactions of an acyl-CoA promiscuous trans-acyltransferase. Chem. Biol. 21, 636-646 (2014).

583 27. Pulsawat, N., Kitani, S., \& Nihira, T. Characterization of biosynthetic gene cluster for the 584 production of virginiamycin $\mathrm{M}$, a streptogramin type A antibiotic, in Streptomyces virginiae. 585 Gene 393, 31-42 (2007).

587 28. Wong, F.T., Jin, X., Mathews, I.I., Cane, D.E., \& Khosla, C. Structure and mechanism of the 588 trans-acting acyltransferase from the disorazole synthase. Biochemistry 50, 6539-6548 (2011). $5921186(2008)$. 
594 30. Musiol, E.M. et al. Supramolecular templating in kirromycin biosynthesis: the 595 acyltransferase KirCII loads ethylmalonyl-CoA extender onto a specific ACP of the trans-AT 596 PKS. Chem. Biol. 18, 438-444 (2011).

597

598 31. Brignole, E.J., Smith, S., Asturias, F.J. Conformational flexibility of metazoan fatty acid 599 synthase enables catalysis. Nat. Struct. Mol. Biol. 16, 190-197 (2009).

600

601 32. Davison, J. et al. Insights into the function of trans-acyl transferase polyketide synthases 602 from the SAXS structure of a complete module. Chem. Sci. 5, 3081-3095 (2014)

603

604 33. Gay, G., Wagner, D., Keatinge-Clay, A.T., \& Gay, D.C. Rapid modification of the pET-28 605 expression vector for ligation-independent cloning using homologous recombination in 606 Saccharomyces cerevisiae. Plasmid 76, 66-71 (2014).

607 


\section{Trans - AT PKS}

(PksX, Bacillus subtilis)

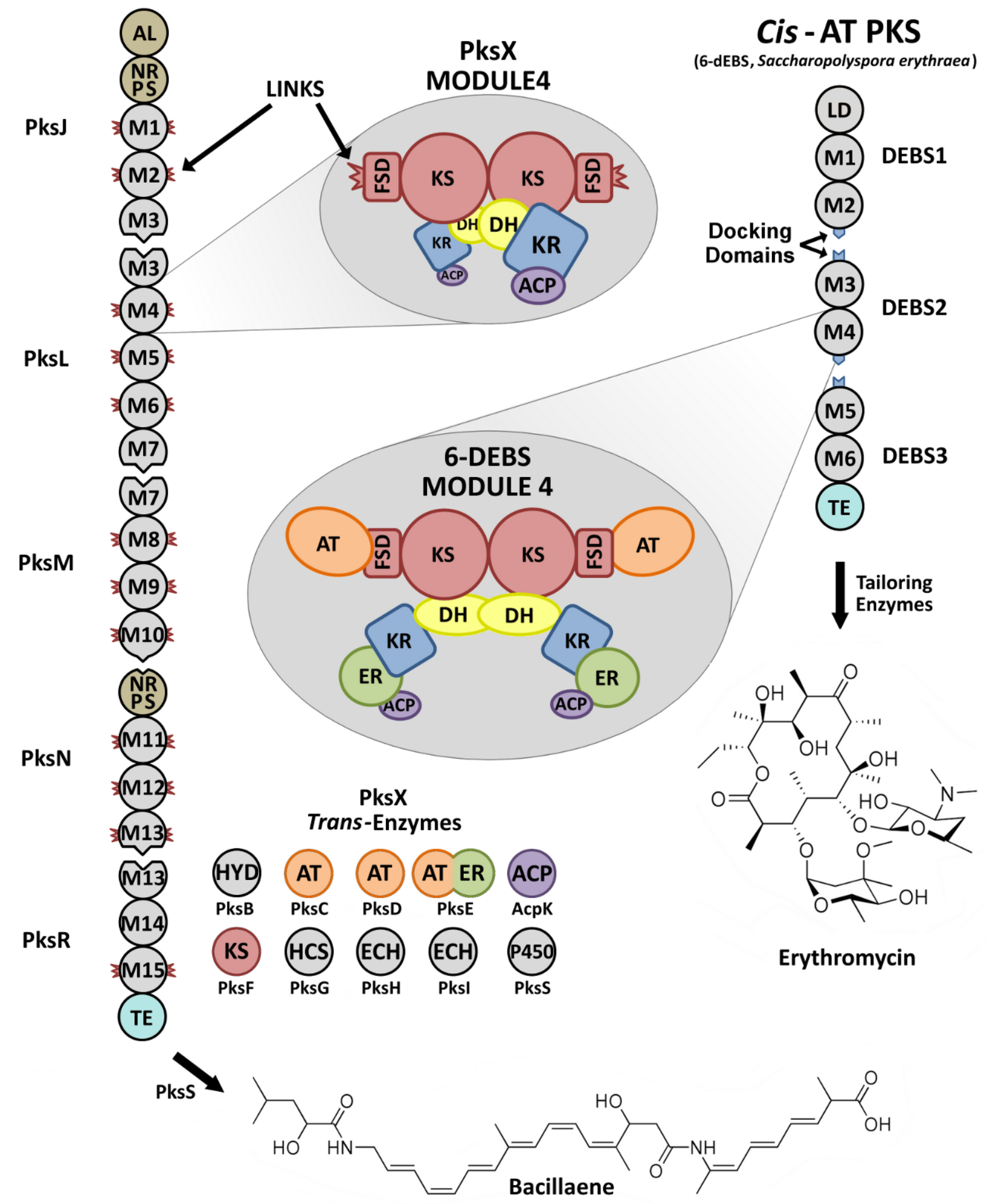



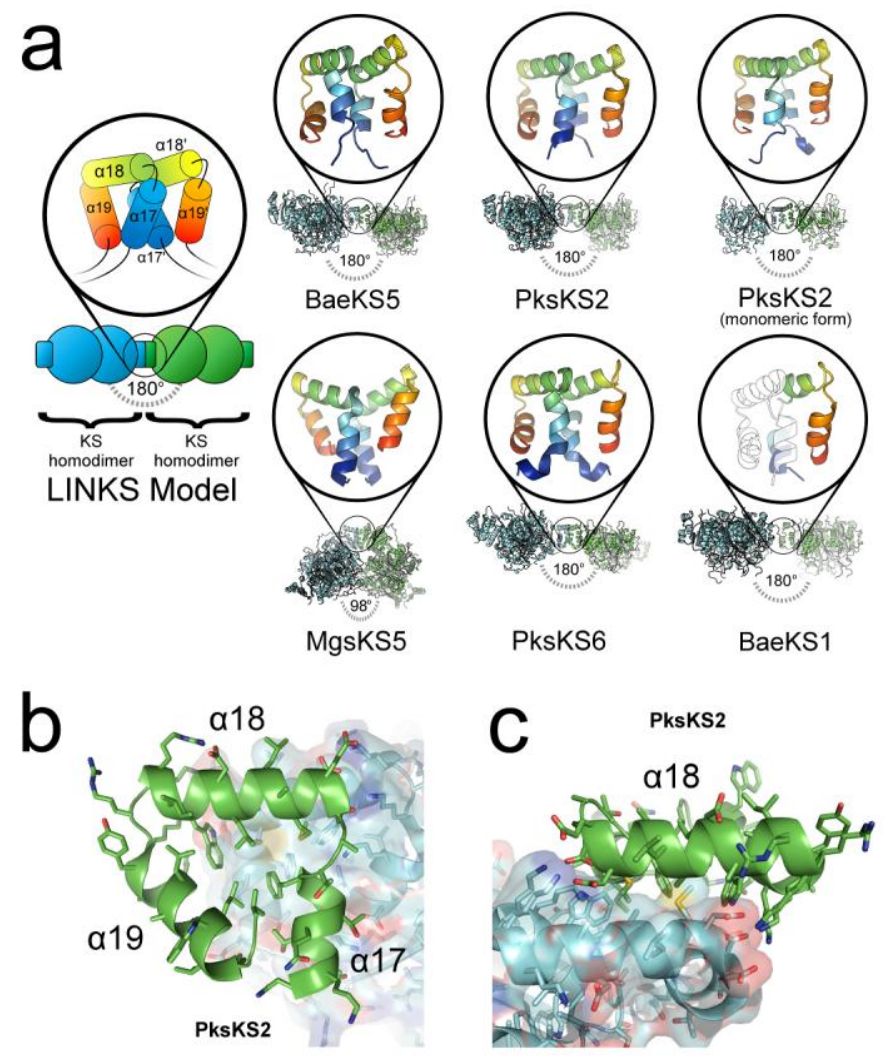

610

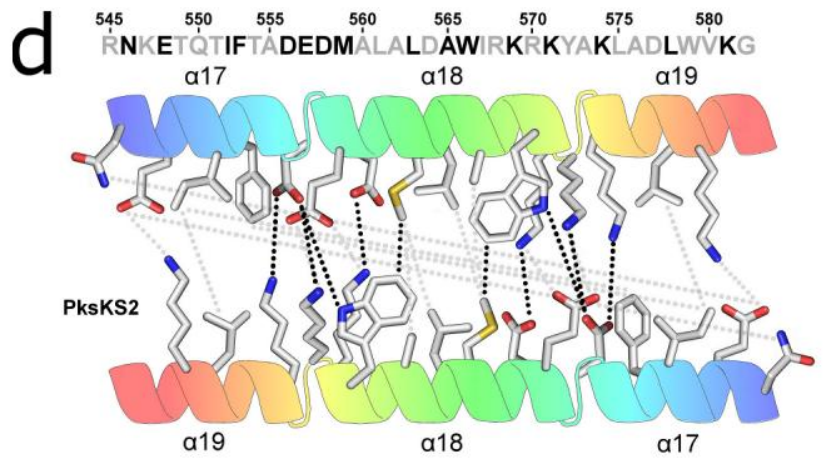

611 
Anionic

$\square$ Cationic

$\square$ Hydrophobic

$\alpha 17$

$\alpha 18$

$\alpha 19$

BaeKS1 (5ELP) - - AVT - GCFRGEKOQAK - - TIAWL * BaeKS1 (SELP) - - A AVT - GCFRGEKQQAK - - - DIAWLS $\begin{array}{llll}\text { PksKS2 } & \text { (4NA1) - - ETDE - - FFRGQSHRN - - - KETQT IFTA } \\ \text { BaeKS5 (5ERB) - - SIDQ - - LYYRGQVKQN - - - KDTMALFTA }\end{array}$

$\begin{array}{lll}\text { BaeKS5 } & \text { (5ERB) - - SIDQ - - L LRGQVKQN - - - KDTMALFTADE } \\ \text { PksKS6 (5ERF) - - NAS - . IFAAHVKTK - . KSEIKLFETDH }\end{array}$

MgSKS7 (4TKT) - SGLPAG - . - AYFNPGGDR - GGAVKDFLT

MgsKS5 (4TL2) S - - - - EAEAEVPWSRGRVRATRETLAALAEK

BaeKS9

- SAG - . - LHRDGTEEN - . - KRMLAVFSAD

BaeKS2 - - NIAG - - LYTGQSHRN . . - KDTFALFTA

GUKS5 - -DSFD - - LYRGGAKRD - . - KETFSQFA I

GUKS11 - -DIED - - - LYKGNVKRD - . - NDSAWL YAVD

GUKS10

BaeKS11

- DLEH. - - LHKGHVKRDRD- - NDSAWLYAV

- -DIEG - - - LSMGQVKRD - - - NDMLAVFTAD

TaKS10

- - HAD - - - LYRGRID - - - - KGTLQMLTEDE

GUKS8

- - LADS - - - VYRGHVKRH - - - KEFLSVFADDA

- - GIEK - - LYRGQVKPN - - - KEALSVFSM

- - AGDA - - - VFRGHVKRN - - - KDALSVFTG

- EHAH - . - IWRGRTAKH - . - . HSLAASLTS

DifKS4

DifKS2

- - TGQN - . - FFTGRNKAM - . - - KQLFT EMTIDNA

DifKS3

BaekS6

GUKS4

- - - GEG - - I I YEAH I KTA - . - KKE I KAFETDD

- - - SSG - - - VFSGRVQEG - - - KNEAGLFSSDENG - . SAA - - -VLSGQAKRT - . - AASI SALAA QPKSKGK - RWRGRVERV - . - - SNGL TDALV - ALTSG - - RVQVGSARQD - . - - ENPLVRLLGED

TaKS - TQTEG - - VQQGLVTQEA - - EEQFDTVLGD
SGAQAS - - GVLRGDVRAA - - - DAQHAPAVD

BTKS10

OnnKS5

ChiKs11 - - - -RIS - GCYTGSARHAG - - - - NVLRI LGD A . . . . . . . VLRGRARSD - . . - DRALLPGDD

DszKS10

OnnKS3

DifKS13

ID - - - - DLARGDVGTTP - - AASRLLLDGR

DQIAD - - - CYRGGTVIKQN - . - IETFSSLADED

QIAD - - CYRGTVIKQN - - I IETFSSLAD
NAPH - - LYRGRAKRN - - - HETVAVFESPE MHVEG - . - LYRGRVQRG . . . . . . . .

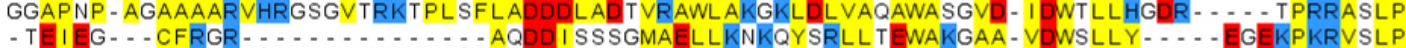

OnnKS6

DifKS11

PedKS10

OnnKS4 - EEPSG - - VYRGDTKAN - - - KAALQE I DDDDRSLEKLIAYFSQDDVHKLAKLWTQGVE - VDWPSLYARMPFAGRSPRRVALP QDGDVPI - YVG - - . NTEDG - AEH I QPLLLRENAARMQEALFAERDLEKMALWWTLGGK - LSWERLYQG. - . - ERVRR I SLP VPGAFRG - . . . - . - DVHGD - KAASLAMDGDDHDREYLERLARDRRLDRLARLWLLGLR - VPWEELH-RDRG - . - RKRVALP

RhiKS11 (4KC5) SSADSKI - FYRFQESDAVQPLESDLN -

DPLAPLLTQWLNGDSQVDWASLYAQP - . . - PVRISLP OzmKS9 (4OPE) P . . . . RVLTGTASRA . . AAP . . . . . . . . . . A AGRTAPELAEAWVRGAP - VAAP . . . . . . AGAPRVSLP 

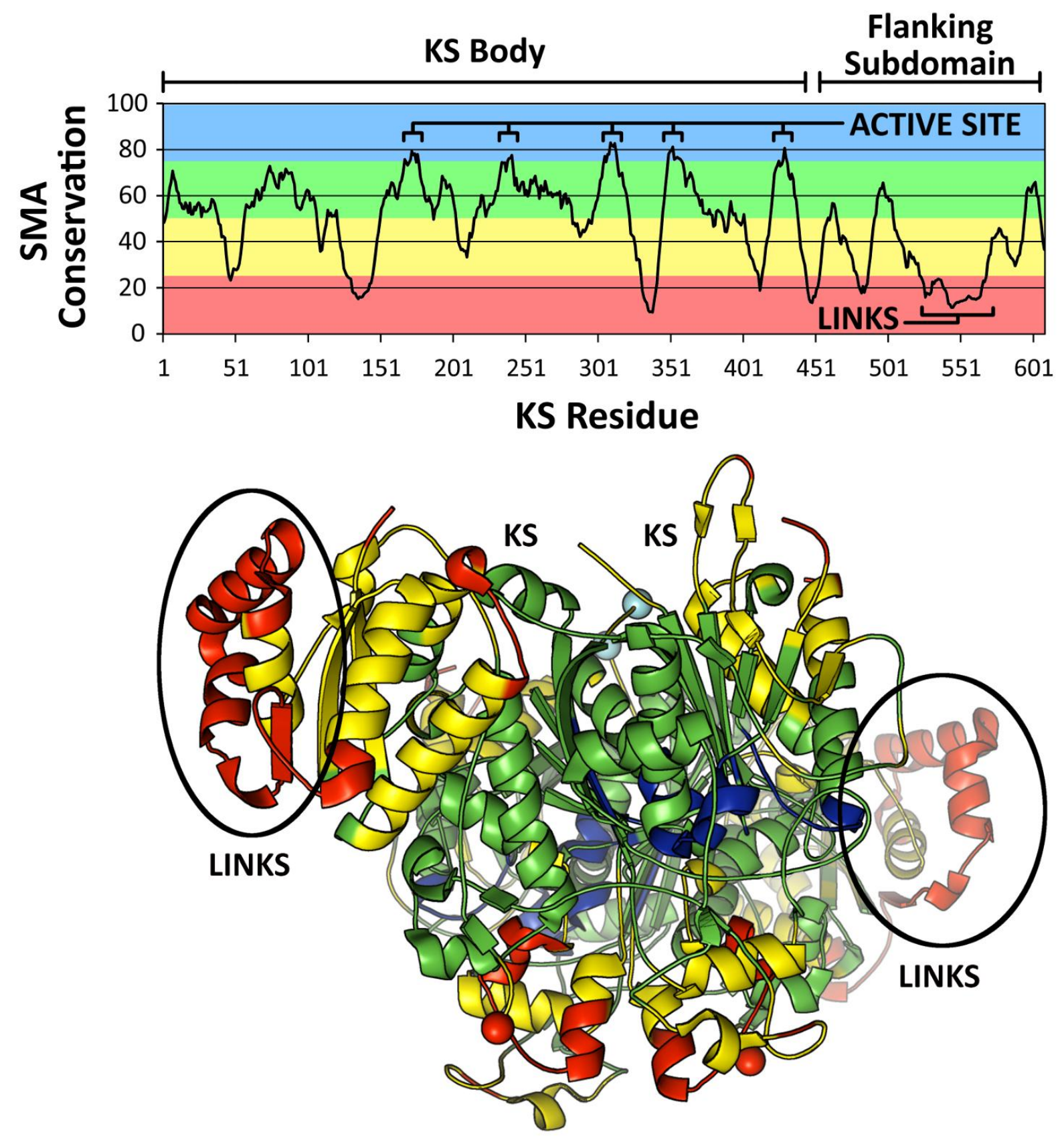

614

615 


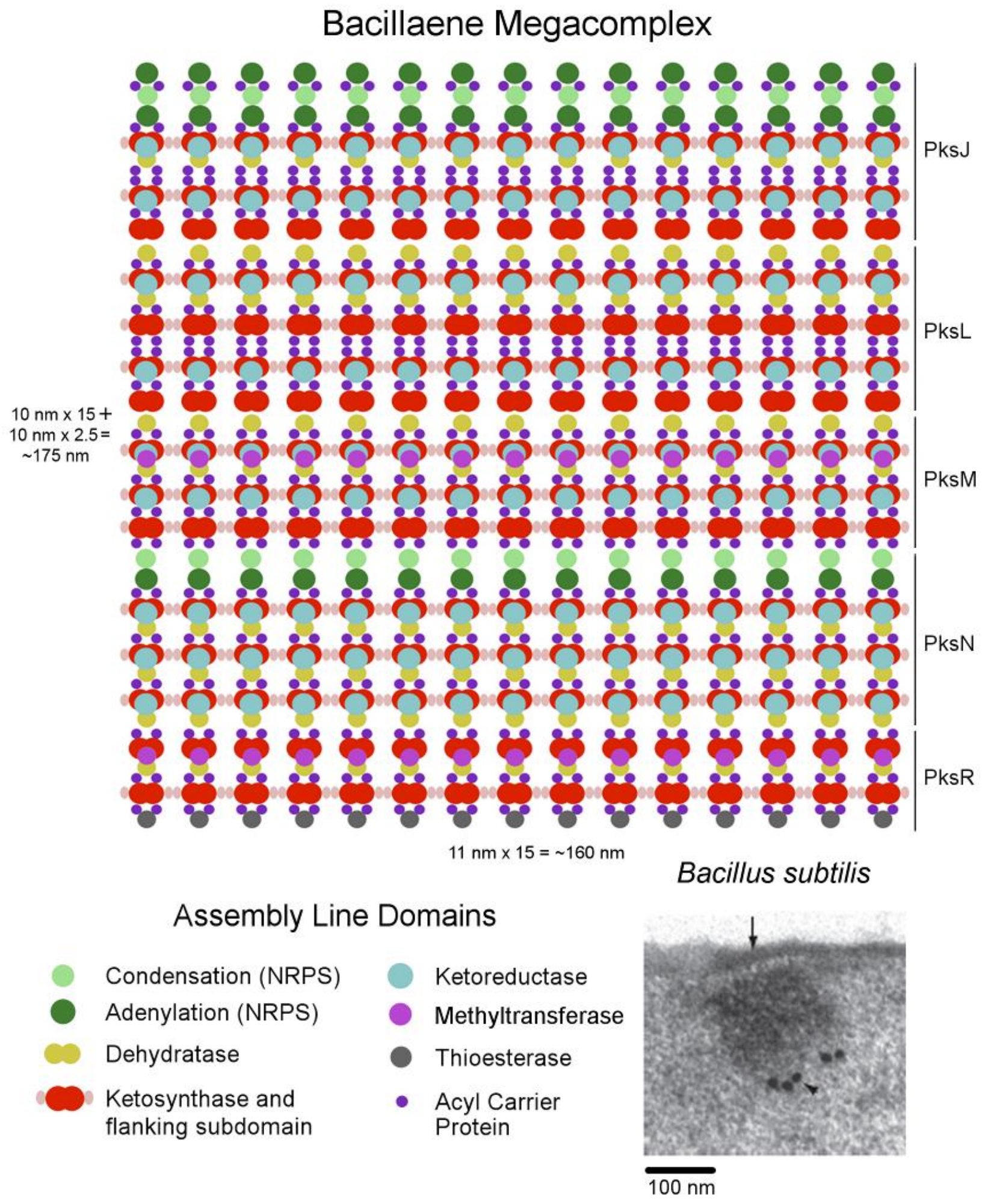

